\title{
Improving Perioperative Data Integrity and Quality via Electronic Medical Record Reconciliation
}

\author{
Jim Ryan \\ Auburn University at Montgomery \\ jryan@aum.edu \\ Sandra Daily \\ Duke University Medical Center \\ sandra.daily@duke.edu
}

\author{
Barbara Doster \\ University of Alabama-Birmingham Hospital \\ bdoster@uabmc.edu \\ Carmen Lewis \\ Troy University \\ cclewis@troy.edu
}

\begin{abstract}
This case study investigates data integrity and quality within the perioperative process via embedded quality control check (QCC) rules, used within a business process management framework to support patient care documentation, performance reporting, patient billing, data analysis, and regulatory agency audits. The study identifies specific perioperative nursing care documentation as electronic medical records and demonstrates how QCC rules, an embedded QCC process, and QCC rule violation reconciliation is applicable to ensuring data integrity and quality within integrated hospital information systems. Based on a 166month longitudinal study of a large 1,157 registered-bed academic medical center, this study provides a priori business process management examples of data integrity and quality within the perioperative process. Recognizing existing limitations, potential capabilities, and the subsequent contextual understanding are contributing factors that yield measured improvement. Theoretical and practical implications and/or limitations of this study's results are also discussed.
\end{abstract}

\section{Introduction}

In the United States, the 2009 American Recovery and Reinvestment Act, the 2010 Affordable Care Act, the Joint Commission on Accreditation of Healthcare Organizations (TJC), and Centers for Medicare \& Medicaid Services (CMS) require performance and clinical outcome reporting as evidence of healthcare provider quality, efficiency, and effectiveness [4]. Consequently, the resulting widespread information systems (IS) and information technology (IT) adoption across United States' hospitals further necessitates the need for value realization [7, 16]. Meeting these demands require hospital administrators and medical professionals alike to leverage IS and IT that yield quality patient care and safety, coupled with efficiency and effectiveness [29].

Within healthcare, a patient's care is the focus of work. Specifically within a hospital's perioperative process, workflow (i.e., surgical patient flow) is driven by operating room (OR) scheduling. To this end, a hospital's perioperative process involves multiple interconnected sub-processes that reflect surgical care for inpatients and outpatients upstream and downstream of OR workflow during pre-assessment, pre-operative, intra-operative, post-operative, and central sterile supply activities. Hence, a hospital's perioperative process is complex [14]. Nonetheless, the perioperative process is one of many core processes nested within the hospital environment that yield overall clinical performance and integrated hospital IS (IHIS) document patient care and clinical outcomes associated with these core processes. The perioperative sub-process activities' and other core hospital sub-process documentation in the IHIS also provides evidence for regulatory agencies (e.g., TJC and CMS) and third party payers, as well as internal performance reporting and data to support operational improvement efforts through business process management (BPM). To this end, IHIS must maintain indisputable data integrity (e.g., validity and consistency) and quality (e.g., completeness and timeliness).

This research investigates how embedded quality control check (QCC) rules, used within a BPM framework, ensure perioperative data integrity and quality for: (1) patient care documentation, (2) performance reporting, (3) patient billing, (4) perioperative data analysis, and (5) regulatory agency audits. The investigation method covers a longitudinal study of a specific IHIS type - a integrated clinical scheduling IS (CSIS) implementation, integration, and use. The resulting systematic analysis and subsequent contextual understanding of the perioperative subprocesses coupled to the integrated CSIS yielded opportunity for measurement and ultimately improvement in perioperative data integrity and quality.

This paper prescribes an a priori approach for embedding integrity and quality as QCC rules into perioperative process data via cross-checking contents of real-time perioperative electronic medical records (EMRs) when surgical cases are completed (e.g., closed). The following sections review previous literature on data quality versus data integrity, BPM, key performance 
indicators (KPIs), perioperative patient care, as well as CMS quality standards and reimbursements. Following the literature review, we present our methodology, case background, observed effects, and results. The conclusion addresses study implications and limitations.

\section{Literature Review}

Integrated IS offer continuity through information sharing and synergy [20], where IS integration is an attempt toward improvement [42]. Likewise, IHIS provide measurement data and subsequent accountability for healthcare quality and cost, creating a dichotomy (e.g., quality versus cost) that represents the foundation for healthcare improvement [11]. To this end, using IHIS data as a resource for accountability and decisionmaking increases the importance for indisputable data integrity and quality.

\subsection{Data Integrity versus Data Quality}

Traditionally, data integrity is the validation of existing correctness or congruence between fields of data stored in a database [15]. This definition differentiates data integrity from quality, where data integrity concerns stored data while data quality relates to data fit for usehow data models the real world [37]. Nonetheless, data integrity issues can lead to poor data quality, while data integrity alone may not correct poor data quality. Furthermore, poor data integrity and/or poor data quality within IS yield flawed information, which leads to flawed decision-making and flawed decisions. Lee \& Strong [21] and later Weiskopf and Weng [48] suggest high data quality yields accurate, complete, accessible, timely, and relevant information. Lee and Strong [21] empirically identified knowledge as an important prerequisite for producing high data quality. To this end, integrity maintaining mechanisms can be embedded within IS processes as routines or within databases as triggers or stored procedures. Lee et al. [22] recommends embedding data integrity and quality within the process as a continuous data quality improvement, dynamic in nature to address changes in business processes similar to the concept of BPM.

\subsection{Business Process Management (BPM)}

Continuous process improvement (CPI) is a systematic approach toward understanding process capability, customers' needs, and sources of observed variation. Tenner and DeToro [39] views CPI as an organizational response to an acute crisis, a chronic problem, or an internal driver. CPI encourages bottomup communication at the day-to-day operations level and requires process data comparisons to control metrics. Incremental improvement gains occur via iterative cycles of analysis, evaluation, and synthesis or plan-do-studyact [43] to minimize observed variation.
This study uses the BPM definition provided by Jeston and Nelis [18, p. 10] as "the achievement of an organization's objectives through the improvement, management, and control of essential business processes." The authors further elaborate that process management and analysis is integral to BPM, where there is no finish line for improvement. Hence, this study views BPM as an organizational commitment to consistent and iterative business process performance improvement that meets organizational objectives. Business analytics is the body of knowledge identified with technology solutions that incorporate definition and delivery of business metrics, performance dashboard management, as well as data visualization and data mining [40]. Business analytics within BPM focus on the effective use of organizational data and information to drive positive business action [18]. The effective use of business analytics demands knowledge and skills from subject matter experts and knowledge workers. Similarly, Wears and Berg [46] concur that IS and/or IT only yield high-quality healthcare when the use patterns are tailored to knowledge workers and their environment. Therefore, BPM success has a strong dependence on stakeholders' contextual understanding of end-to-end core business processes [18], where poor data integrity or quality distort stakeholder understanding.

\subsection{Key Performance Indicators (KPIs)}

Performance measurement is essential for purposeful BPM, as information before and after the intervention is integral to process improvement. Performance measurement also demands data integrity and quality to minimize bias and impart stakeholder trust. Relatedly, Ackoff [0] proposed feedback within IS design as an embedded control to avoid management misinformation. Likewise, Lee et al. [22] views data integrity and quality as dynamic process-embedded feedback. Similarly, organizations define data metrics as KPIs to assist management via IS feedback in monitoring organizational action via business processes $[24,30,51]$. For example, OR schedules are tightly coupled to individual OR suites, patients, and surgeons. When pre-operative tasks are incomplete or surgical supplies/instruments/devices or personnel are not available at time of surgery, the scheduled case and subsequent scheduled cases for the particular OR suite or surgeon are delayed. Perioperative delays risk patient safety and care.

Operational and tactical KPIs in perioperative subprocesses are numerous, but intra-operative KPIs should include: (1) monitoring the percentage of surgical cases that start on-time (OTS) or first-of-the-day surgical case on-time starts (FCOTS), (2) OR turn-around time (TAT) between cases, (3) OR utilization (UTIL), and (4) labor hours per patient care hours as units-of-service (UOS) expended [17, 19, 27, 49]. Tarantino [38] noted how lower OR TAT and a flexible work environment are 
critical success factors (CSFs) for physician satisfaction, which in turn is a CSF for hospital margin. In contrast, inefficient and ineffective processes yield poor operational and tactical KPI metrics (i.e., OTS, TAT, UOS, or UTIL) that affect strategic CSFs of patient safety, patient quality of care, surgeon/staff/patient satisfaction, and hospital margin [23].

\subsection{Perioperative Patient Care}

Within the perioperative process, specialized physicians (e.g., surgeons and anesthesiologists), nurses, and staff provide pre-assessment, pre-operative, intraoperative, and immediate post-operative patient care. Hence, perioperative patient care occurs via teamwork with specific roles and activities that require awareness, communication, and coordination among different members who may not meet face-to-face. Surgeons evaluate, prescribe, and perform the surgical procedure. Anesthesiologists evaluate, prescribe, and administer anesthesia [2]. Nurses evaluate, assist physicians, provide either ambulatory or acute care per physicians' instructions, as well as monitor and document patient care within the IHIS. Perioperative staffs facilitate location, supplies, instruments, and equipment per physician instructions. As a result, perioperative care yields patient end-state goals where: (1) a correct diagnosis for surgical intervention is identified with noted co-morbidities and patient consent; (2) a patient undergoes the surgical procedure; (3) a patient exhibits minimal exacerbation of existing disorders; (4) a patient avoids new morbidities; and (5) a patient experiences prompt procedure recovery [36].

Workflow complexity is a barrier to perioperative patient end-state goals [14]. Numerous issues can arise within perioperative sub-processes that place a patient's end state goals at risk, which include: (a) inaccurate and/or incomplete patient care documentation [12, 35]; (b) hospital-acquired-conditions or hospital-acquiredinfections (HACs or HAIs) connected with negative financial incentives [8, 25, 44]; (c) emergency surgery patients [47]; (d) intensive care surgery patients with unplanned discharges [41]; (e) or nurse-staffing shortages [1] to identify a few. However, perioperative best practices minimize risk while supporting and insuring patient end-state goals. For example, accurate and complete nursing documentation is essential to communicate and coordinate subsequent patient care downstream [28]. Pre-operative integrated evaluations communicate and document practitioner-patient awareness to avoid conflicts and identify potential OR specific risks [36]. Similarly, computerized provider order entry (CPOE) communicates, coordinates, and documents provider prescribed patient care to improve patient outcomes [31]. Data integrity and quality within the CSIS ensures patient's diagnosis, physician orders, events, experiences, and outcomes are correctly documented as perioperative care.

\subsection{CMS Standards and Reimbursements}

CMS requires hospitals that receive CMS patient care reimbursement to submit quality of care outcome measures quarterly, which have evolved over time $[6,7$, 16]. In 2005, CMS began encouraging improvements in Medicare patients' quality of care via pay-forperformance (P4P) as a CMS payment model that rewards healthcare providers for meeting certain performance measures in quality and efficiency [5]. In addition to $\mathrm{P} 4 \mathrm{P}, \mathrm{CMS}$ includes disincentives of reducing reimbursements [8, 44] for negative consequences of care that should never occur, defined by the National Quality Forum (NQF), including hospital infections under the surgical care improvement project (SCIP) [25].

The Health Insurance Portability and Accountability Act (HIPAA) of 1996 revised the United States Social Security Act and established the Medicare Integrity Program within CMS to deter fraud and abuse associated with healthcare provider reimbursement [9]. The Medicare Integrity Program authorized CMS to outsource the auditing of healthcare provider reimbursement to third parties or recovery audit contractors (RACs). The resulting RAC program is an integral part of CMS" "benefit integrity" efforts, which is responsible for highlighting common billing errors, trends, and other CMS overpayment or underpayment issues [9].

In fiscal 2014, CMS RACs collectively identified and corrected $\$ 2.57$ billion in improper payments to healthcare providers [9]. The CMS RAC correction amount in fiscal 2015 decreased by $82.8 \%$ due to a prohibition by the United States Congress on CMS RACs performing patient status reviews until healthcare providers could fully comprehend the new CMS Inpatient Prospective Payment System Final Rule [9]. The prohibition on CMS RACs performing patient status reviews ended in October 2015 and contracts from CMS to regional RACs were awarded in November 2016. Common billing errors and CMS under or over payments can identify data integrity and quality issues within a hospital's IHIS that can initiate RAC audits, which will delay and/or disallow CMS reimbursements [9]. Irreconcilable data integrity and quality issues discovered in RAC audits can be construed as fraud and passed on to the United States Department of Justice or Office of the Inspector General [9]. Ensuring the data integrity and quality of healthcare reimbursement claims to CMS is a CSF for any healthcare provider.

\section{Research Methodology}

The objective of this study is to examine how embedded data quality control checks, used with a BPM framework, ensure perioperative data integrity and data quality for downstream data consumers, knowledge workers and stakeholders. To this end, case research is particularly appropriate $[13,50]$. Paré [26] recommends 
using a positivist case study methodology in IS research and operationalizing positivist criteria supports quality case research [3]. Another advantage of the positivist approach [45] to case research allows concentrating on a specific hospital service in a natural setting to analyze the associated qualitative problems and environmental complexity. Hence, our study took an in-depth case research approach.

Our research site (e.g. University Hospital) is an academic medical center, licensed for 1,157 beds and located in the southeastern United States. University Hospital is a Level 1 Trauma Center, with a robotics program across eight surgical specialties as well as a Women's/Infant facility. University Hospital's recognition includes Magnet [1] since 2002 and a Top 100 Hospital by U.S. News and World Report since 2005. Concentrating on one research site facilitated the research investigation and allowed collection of longitudinal data. This research spans activities from August 2003 through May 2017, with particular historical data since 1993. During the 166-month study, we conducted field research and collected data via multiple sources including interviews, field surveys, site observations, field notes, archival records, and document reviews.

\section{Case Background}

Perioperative Services (UHPS) is the University Hospital department designated to coordinate and manage perioperative patient care across Pre-admissions, Admissions, Surgical Preparations (PRE-OP), Central Sterile Supply (CSS), OR Surgery and Endoscopy, and Post Anesthesia Care Units (PACU). The workflow through CSS reprocesses all reusable surgical instruments/devices and moves supplies to pre-operative, intra-operative, and post-operative activities. The following sections highlight tools, events, and outcomes that have shaped UHPS' BPM approach.

\subsection{CSIS Implementation}

UHPS implemented a new, agile CSIS in 2003, after using its prior CSIS for 10 years. The new CSIS supports OLAP tools, a proprietary structured query language, and both operational and managerial data stores (i.e., an operational database and a separate perioperative data mart). Flexible routing templates or surgical preference cards (SPCs) allow standardization of surgical care data (i.e., particular supplies and instruments needed) or SPC customization for specific surgeons and/or procedures. Since the CSIS implementation, over 7,750 generic and custom SPC configurations facilitate the surgical specialty services (SSS) represented in Table-1. Similarly, the agile CSIS data marts serve as the central repository for perioperative process data used to support improvement initiatives as well as report KPIs with a business intelligence layer to support data visualization.

Table 1 - Current CSIS SPCs

\begin{tabular}{|l|r|}
\hline Surgical Specialty Service & SPCs \\
\hline BURN - Trauma burns & 26 \\
CARDIO -Cardiovascular \& Thoracic & 946 \\
ENT - Ear, Nose, \& Throat & 1,030 \\
GI - Gastro-intestinal & 460 \\
GYN - Obstetrics, oncology, incontinence & 611 \\
NEURO - Neurological & 763 \\
ORAL - Oral Maxilla Facial & 236 \\
ORTHO - Orthopedic, joint/device & 1,208 \\
PLAS - Plastic surgery & 681 \\
SURG ONC - Surgical oncology & 329 \\
TX - Transplants (liver, renal) & 194 \\
TRAUMA - Trauma, MASH & 203 \\
URO - Urology & 533 \\
VASCULAR - arteries \& blood vessels & 558 \\
\hline
\end{tabular}

\subsection{November 2004}

University Hospital opened a new diagnostic and surgical facility (e.g. North Pavilion) in November 2004. UHPS relocated CSS onto one floor (e.g. 3rd) with PreOP, ORs, and PACU on each of the two floors above. The new facility expanded UHPS to cover an additional floor and nine ORs (i.e., 33\% capacity increase) for a total of 40 state-of-the-art OR suites, each having standardized and surgical specific equipment. Within six weeks of occupancy, a scheduling KPI reflected chaos. Surgical OTS plunged to $18 \%$ during December 2004. Having only $18 \%$ OTS is unacceptable in a highly competitive hospital industry, as $82 \%$ of scheduled surgeries experience delays and risk patient care and safety.

In January 2005, UHPS expressed concerns before a quickly convened meeting of c-level, nursing, and physician representatives. The meeting yielded a hybrid management structure and governance in the formation of a multidisciplinary executive team, chartered and empowered to evoke change. The executive team consisted of perioperative stakeholders (i.e., surgeons, anesthesiologists, nurses, and UHPS). The executive team's charter was to focus on patient care and safety, attack difficult questions, and remove inefficiencies. No issue was off-limits.

\subsection{Perioperative Improvement and BPM}

University Hospital launched a process improvement effort in 2005 to address the perioperative crisis. This CPI effort resulted in the executive team enlisting numerous task forces to address specific opportunities, which was the foundation for their current 
BPM approach. Since 2005, UHPS has focused datadriven analysis of KPIs to gauge process variance, identify improvement opportunities from variances, and improve end-to-end workflow [32]. Using this systematic BPM approach, UHPS implemented numerous improvement efforts across each of the perioperative sub-processes (e.g., pre-assessment, PreOP, OR, PACU, and CSS) to improve and balance patient workflow [33].

In 2009, UHPS expanded its management beyond the initial 32 general ORs (GENOR) and 8 cardiovascular OR suites (CVOR) within the North Pavilion campus to the other campuses of University Hospital Health System (UHHS) including 16 OR suites at the Highland campus (HHOR) and 8 endoscopy labs at the TK Clinic campus. In 2011, UHPS also developed a preoperative assessment, consultation, and treatment (PACT) clinic to manage all pre-operative patient flow into UHHS. Two additional general ORs have been equipped since 2013 at the North Pavilion campus to bring UHPS management to 58 ORs and 11 endoscopy labs. Overall, UHHS has experienced a $10.9 \%$ increase in surgical cases since 2007 with $59 \%$ of the average case volume being in-patient and $41 \%$ being out-patient. Emergency surgeries account for $5.3 \%$ of the average case volume with $94.7 \%$ being routine.

\section{Observed Effects}

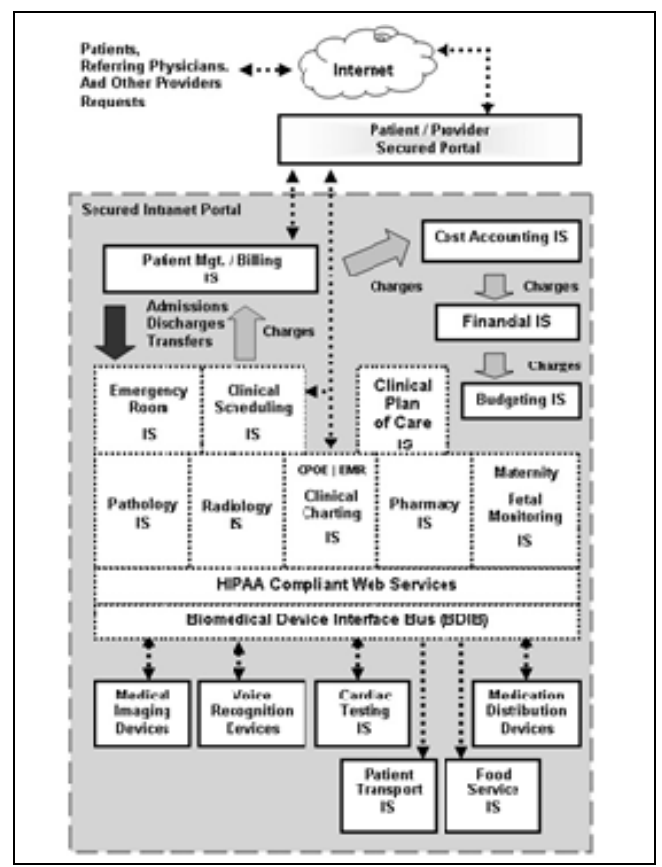

Figure 1 - UHHS Integrated Hospital IS

The IHIS depicted in Figure-1 depicts how the integrated CSIS schedules, records, and facilitates perioperative workflow across UHHS, where the CSIS coordinates, facilitates, and documents perioperative patient care and outcomes [33]. Integration of the IS depicted in Figure-1 occur with either bidirectional data exchange or unidirectional for limited exchange. The seven IS modules clustered around the CSIS directly support and extend the CSIS suite, where the Clinical Charting IS houses CPOE and EMRs. The HIPAA compliant Web services and biomedical device interface bus (BDIB) integrate ancillary IS, clinical data sensors, and bio-medical equipment. The institutional intranet serves as a single entry secured portal to extend each IS according to particular user rights and privileges negotiated via user authentication.

UHHS surgical admissions occur via UHHS physician referrals, non-UHHS physician referrals, and patients seeking emergency treatment. All medical records (i.e., in-patient or out-patient), admissions, diagnostics, clinical data, observations, as well as discharges occur via the same IHIS. All perioperative material supplies, medical devices, and labor charges captured from surgical patients' are documented in the CSIS. Charges flow from the CSIS through to Cost Accounting, Financial, and Budgeting IS. Hence, high data integrity and quality are inherent requirements.

\subsection{Perioperative Documentation as EMRs}

Recorded and documented within the integrated CSIS, surgical UHHS patients move through the perioperative workflow via events: (1) A clinic visit resulting in surgery scheduling, (2) PACT Clinic evaluation, (3) day of surgery admission, (4) PreOP, (5) Intra-operative or Endoscopy procedure, (6) PACU, (7) PACU Phase-II, and (8) discharge or movement to a medical bed. Each perioperative event creates an ambulatory EMR associated with the patient's unique medical record (MRN), encounter, and CSIS case number (i.e., these provide unique surgical case tracking IDs). UHPS nurses record patient care details into the CSIS as EMRs to manage and document patient care across perioperative workflow. Table- 2 is the current UHHS listing of CSIS nursing documentation EMRs that includes the fiscal implementation year of UOS charge capture, UOS standard, UOS unit, and associated perioperative sub-process [33].

UOS standards reflect UHPS labor associated with particular patient care activities represented in each EMR - one hour of patient care time, an Endoscopy procedure, or a sterilized instrument load. Aggregate UOS metrics reflect patient care hours by each perioperative sub-process. Nursing documentation EMRs from the CSIS generate charges for perioperative care into the individual patient's billing account and credits UHPS for labor and material costs expended.

UHPS implemented automated quality control check (QCC) rules between nursing EMRs in October 2016 to improve data integrity and quality with respect to completeness, context, time, location, nurse, and surgeon. The CSIS QCC rule validation process and corresponding BPM approach to EMR reconciliation 
ensures integrity and quality for perioperative data applications downstream to data consumers, knowledge workers, and other stakeholders. The following sections detail observed effects of the CSIS QCC rules, the CSIS embedded QCC rule process, and the non-finalized EMR reconciliation.

Table 2 - CSIS Nursing EMRs and UOS

\begin{tabular}{|c|c|c|c|c|}
\hline $\begin{array}{c}\text { Sub- } \\
\text { process }\end{array}$ & $\begin{array}{c}\text { Perioperative } \\
\text { Nursing EMR }\end{array}$ & FY & $\begin{array}{c}\text { UOS } \\
\text { Std. }\end{array}$ & $\begin{array}{c}\text { UOS } \\
\text { Unit }\end{array}$ \\
\hline Admissions & $\begin{array}{c}\text { Ancillary } \\
\text { Services | Family }\end{array}$ & 2007 & -- & -- \\
\hline PACT & $\begin{array}{c}\text { PreOP Nursing } \\
\text { Assessment }\end{array}$ & 2012 & 1.93 & Hrs. \\
\hline PreOP & $\begin{array}{c}\text { Endo PreOP } \\
\text { Nursing }\end{array}$ & 2014 & -- & Proc. \\
\hline PreOP & $\begin{array}{c}\text { Endo Sedation } \\
\text { Nursing }\end{array}$ & 2014 & 2.1 & Hrs. \\
\hline PreOP & $\begin{array}{c}\text { Regional Block } \\
\text { Nursing }\end{array}$ & 2014 & 2.21 & Hrs. \\
\hline CSS & $\begin{array}{c}\text { CSS Sterilized } \\
\text { Instruments }\end{array}$ & 2003 & 3.52 & Load \\
\hline OR & $\begin{array}{c}\text { OR Nursing | } \\
\text { CVOR }\end{array}$ & 2007 & 9.04 & Hrs. \\
\hline OR & $\begin{array}{c}\text { OR Nursing | } \\
\text { Cardiac } \\
\text { Perfusion }\end{array}$ & 2012 & 4.22 & Hrs. \\
\hline OR & $\begin{array}{c}\text { OR Nursing | } \\
\text { GENOR - HHOR }\end{array}$ & 2003 & 7.45 & Hrs. \\
\hline OR & $\begin{array}{c}\text { OR Nursing | } \\
\text { ENDO }\end{array}$ & 2014 & 6.92 & Proc. \\
\hline PACU & $\begin{array}{c}\text { Ancillary } \\
\text { Services | Room } \\
\text { Cleanup }\end{array}$ & 2005 & -- & Hrs. \\
\hline PACU Overflow & 2014 & 2.71 & Hrs. \\
\hline $\begin{array}{c}\text { PACU Phase-II } \\
\text { Nursing }\end{array}$ & 2014 & 1.93 & Hrs. \\
\hline
\end{tabular}

\subsection{CSIS Quality Control Check (QCC) Rules}

Data integrity solutions require flexible logic control capabilities while performing numerous types of validation [10]. To this end, UHHS perioperative data collected via CSIS nursing documentation EMRs have source entry referential integrity checks to offer specific valid domain options through drop-down boxes, radio buttons, or check boxes. Default information from the GENOR, CVOR, HHOR, and ENDO schedules are prepopulated into data fields as default selections and edit checks can be performed across data fields when an EMR is submitted. However not all valid options are correct. Furthermore, the 14 EMRs listed in Table-2 occur at different times, in different locations, in different sequences. Hence the need for flexible logic control, across numerous types of validation, for as many as 6 EMRs in sequence. As a result, UHPS developed
QCC rules as data logic rules, which evolved to identify potential rule violations as documented by perioperative staff. The current list consists of 48 rules to monitor EMR completeness, context, time sequences, location, nurse, and surgeon. Table-3 summarizes the current 48 QCC rules by EMR type.

Table 3 - QCC Rules by EMR Type

\begin{tabular}{|c|c|c|}
\hline EMR & Check IDs & \# of QCC Rules \\
\hline PACT & 1005 & 1- PACT diagnosis \\
\hline \multirow{3}{*}{ PreOP } & 102 & 1- patient in PreOP \\
& 1001 to 1004 & 4- PreOP time sequence \\
& 1006 & 1- PreOP nurse ID \\
\hline \multirow{3}{*}{ OR } & 103 & 1- patient \& start in OR \\
& 2001 to & 11- OR time sequence \\
& 2018 & 7- OR specific content \\
& 5001,5002 & 2- overlap or - turn time \\
\hline \multirow{3}{*}{ PACU } & 104 to 106 & 3-PACU completeness \\
& 3001 to & 11- PACU time sequence \\
& 3013 & 2-PACU nurse/surgeon \\
\hline \multirow{2}{*}{ All } & 101 & 1- case completeness \\
& 107 to 109 & 3- key completeness \\
\hline
\end{tabular}

The 48 QCC rules described in Table 3 are maintained in a SQL database and screen EMRs for completeness and congruity. Each QCC rule has a corresponding SQL procedure that performs a specific validation check for EMRs in the specific perioperative sub-process. The QCC logic rule \#2011 validates Ancillary Services | Room Cleanup EMRs in the OR intra-operative sub-process where completed case cleanup times range between 5 to 60 minutes. If the Ancillary Services | Room Cleanup EMR is short (less than 5 minutes) or excessive (greater than 60 minutes), then the OR EMR is flagged for exception review. Calculations on the OR cleanup time metric yield the intra-operative TAT KPI (e.g., OR turnaround time). An example of this specific data QCC rule logic is:

\#2011 - If the total cleanup minutes (CLEANUP_STOP_T minus CLEANUP_START_T) are less thā 5 minutes or greater than 60 minutes then flag record as inaccurate OR cleanup times.

All data QCC rules, stated similarly to \#2011 above, are housed in a flexible dictionary table within a SQL database where rules can be added or modified as needed. Inactive QCC rules are ignored during the CSIS embedded QCC rule process.

\subsection{CSIS Embedded QCC Rule Process}

Prior to October 2016, OR scheduling nurses manually reconciled completed perioperative nursing documentation EMRs. With the automated CSIS embedded QCC rule process, the OR Analytics Director (i.e., one person) reconciles exceptions when EMRs 
violate QCC rules and OR scheduling nurses now focus on CSIS SPCs, OR scheduling, training, and BPM improvement efforts.

During off-peak times each morning, a CSIS SQL procedure applies the QCC rules across all nursing documentation EMRs of finalized (e.g., completed) surgical cases prior to moving the finalized case EMRs to the data mart. Surgical case EMRs that violate QCC rules become non-finalized with the completed case flag turned off. Finalized case EMRs passing QCC rules are then transported to the perioperative data mart. The QCC rule validation procedure yields an exception report summarizing the number of finalized cases reviewed, the number of non-finalized cases retained, the number of unresolved flags from QCC rule violations, as well as an itemized list by case tracking IDs, QCC ID, QCC rule violation, error comment field, and the nurse responsible for entering the nursing EMR under review. Figure 2 illustrates the CSIS embedded QCC rule process and Table-4 is an excerpt of a reconciled non-finalized case exception report during January 2017. The embedded QCC process prevents any completed case becoming finalized until all QCC rules are met. EMR charge capture depicted in Figure 1 occurs within the IHIS from CSIS triggers on finalized case EMRs that pass the embedded QCC rule validation process.

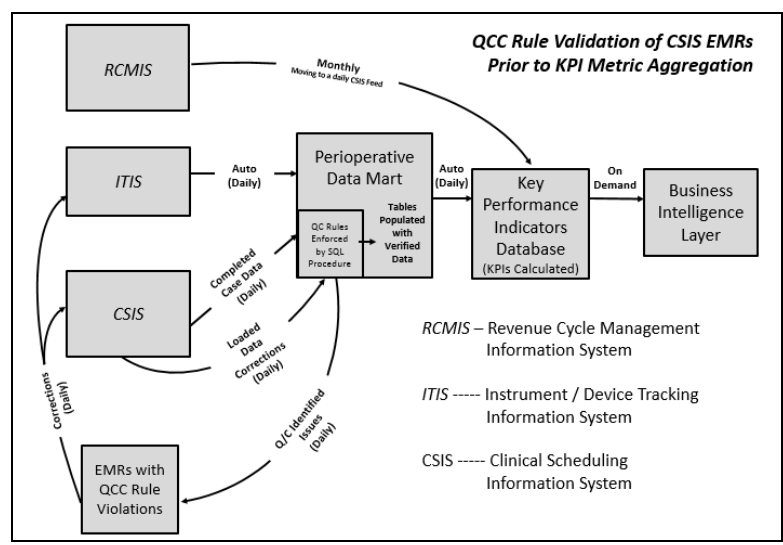

Figure 2 -QCC Rule Validation Process

\subsection{Non-finalized EMR Reconciliation}

The non-finalized case exception report depicted in Table-4 is reconciled daily where each QCC rule violation is reviewed with nursing staff for resolution, the corrected nursing EMR data field information is obtained, and the resolution for the specific error resulting in the rule violation is noted. The reconciled non-finalized case exception reports are archived in Microsoft SharePoint and the corrected EMR data field information is transferred to a Microsoft Access database table for reprocessing. Microsoft Access is used to front-end Microsoft SQL Server where a SQL procedure updates the CSIS with the corrected nursing EMR data field information by MRN, encounter number, and case number key as well as setting the completed case flag to on. The reconciled completed cases are then ready for the embedded CSIS procedure to apply data QCC rules according to the CSIS processing schedule or earlier as needed. The data QCC rules, embedded QCC process, and nursing non-finalized EMR reconciliation in combination support BPM by improving the data integrity and quality of perioperative nursing care EMRs that document perioperative patient care.

Table 4 - QCC Rule Violation Exception Report for January 24, 2017

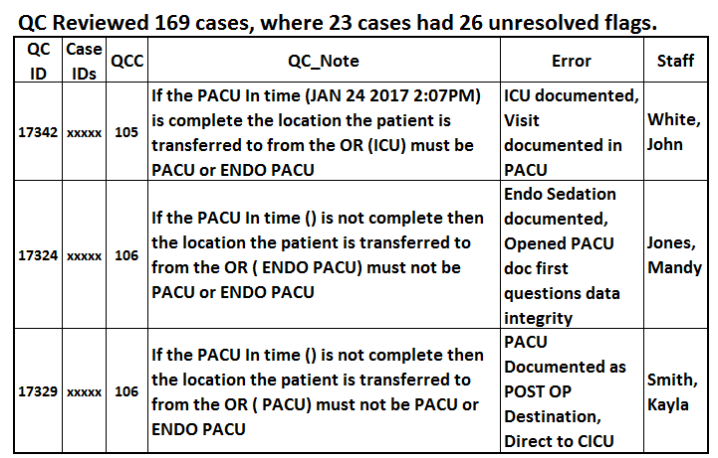

The non-finalized case exception reports in aggregate offer UHPS an opportunity to monitor, improve, and control nursing EMR documentation errors. A wider horizon of review can reflect and target where EMR errors are occurring more frequently, within which perioperative sub-process, and by whom. Focusing on frequency and location allow targeting system changes to address EMR errors that yield the most impact, while focusing on staff that require additional education, training, and control efforts. Figure-3 depicts a Pareto chart of the nursing EMR QCC rule violations by location for a 29 day horizon between January and February 2017. During this time frame, the embedded QCC rule process released $91 \%$ of first time through finalized case nursing EMRs and the 3,538 completed cases passed $99.7 \%$ of the QCC rules.

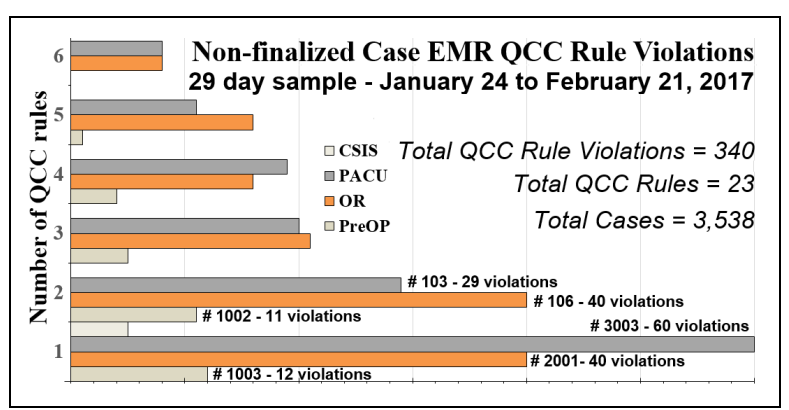

Figure 3 - BPM Approach to EMRs Errors

From the top three QCC rule violations listed on Figure-3 (i.e., depicted at the bottom), we identify that QCC rule \#3003 from post-operative PACU identified the most nursing EMRs errors with QCC rule \#106 and \#2001 from intra-operative OR having the second most. 
The QCC rule \#3003 represents time sequencing for PACU nurse ready. QCC rules \#106 and \#2001 represent PreOP-patient-out to patient-in-OR times do not match followed by time sequence mismatch for patients moved to PACU. Over the three sub-processes during this sample time frame, nursing EMR documentation errors for intra-operative OR (e.g., 153) and post-operative PACU (e.g. 149) represented 89\% of the errors and the top two QCC rule violations across the three sub-processes accounted for $56 \%$ of the errors. Also, no individual nurse accounted for more than $4 \%$ of the errors, where three nurses did have error rates from $3 \%$ to $4 \%$. This type of analysis across the three perioperative sub-processes is available for EMR errors during any given time period since October 2016 using the BPM framework approach.

\section{Brief Discussion and Summary}

UHPS embedded the QCC process depicted in Figure 2 as part of the automated extraction, transfer, and aggregation of KPIs across perioperative sub-processes. Perioperative process complexity [14] broken-down into sub-processes demonstrates a proven approach to understanding complexity by reducing the phenomenon perspective into smaller more manageable and comprehendible units [34]. The embedded QCC process ensures data integrity and quality of sub-process KPIs, extracted from nursing documentation EMRs, to measure process performance and target improvement opportunity. Likewise, the BPM approach to EMR reconciliation allows UHPS to track the cases finalized on first run, the cases requiring EMR reconciliation, investigate the reasons for EMR errors, as well as where to focus improvement and education.

Since the embedded QCC process began in October 2016, the QCC rule design demonstrated flexibility to control numerous types of validation capabilities [10] as the original set of 21 QCC rules increased to 34 during the first two months of use and then to 48 during April 2017, as sumnmarized in Table-3. Figure-4 depicts the percentage of cases finalized on first run, from December 2016 to May 2017, without QCC violations. Through March 2017, the trend was increasing until the addition of 14 new QCC rules in April which identified more EMR exceptions to reconcile. The increase in QCC logic rules assisted in reconciling potential data integrity and quality issues identified on specific subprocess EMRs and by downstream data consumers.

EMRs lacking accurate time sequencing across a completed surgical case is one identifiable highfrequency reconciliation error. Nurses can see via the CSIS the patient scheduled for their OR suite or OR patients nearing transport ready to their PACU site. The error occurs when a nurse opens an EMR early in anticipation of scheduled patient care documentation, prior to the patient arriving in the particular sub-process area. Data integrity and quality issues exist when an
EMR time mismatch occurs, which infers the patient is in two locations at once and labor charges risk overstatement. These issues unreconciled could trigger a RAC audit from a CMS patient status review. Hence, the need for validated EMR time sequences.

Figure-5 depicts the December 2016 to May 2017 results of UHPS' BPM approach to EMR reconciliation exceptions-EMRs opened early or with incomplete data. The percentage of cases with at least one EMR opened early is down from $19.4 \%$ in January to $15.5 \%$ in April. Also, of all the completed surgical cases since December, $6.5 \%$ have had at least one EMR with incomplete data. The embedded QCC rule validation identified each of these EMR exceptions and the EMR reconciliation resolved each rule violation. All of the QCC rule violations in Figure-5 were identified, reconciled, and corrected.

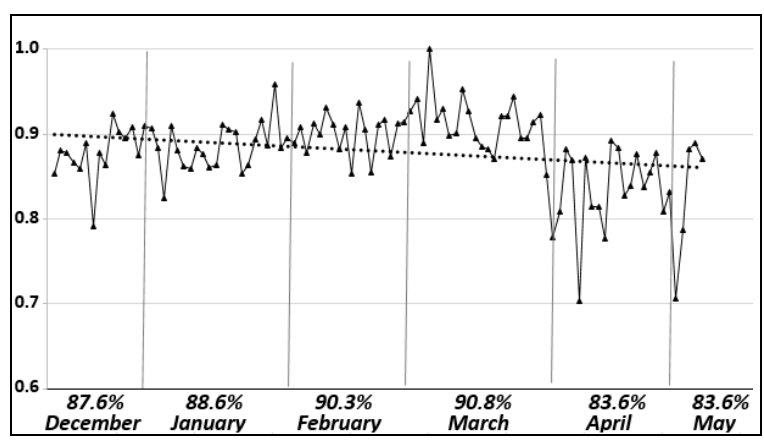

Figure 4 - Finalized Cases on First Run December 2016 to May 2017

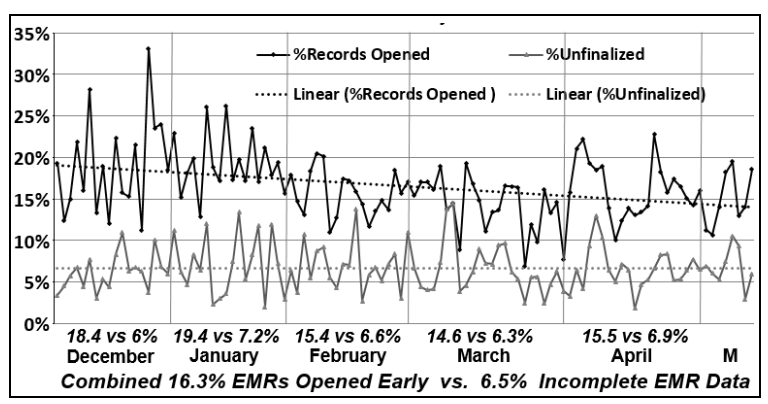

Figure 5 - \% EMR Reconciliation Exceptions December 2016 to May 2017

\section{Conclusion}

Empowered individuals, integrated IS, and a CSIS embedded QCC rule process within a BPM framework allows UHPS to ensure nursing care EMRs within the CSIS meet data integrity and quality standards for: (1) patient care documentation, (2) performance reporting, (3) patient billing, (4) perioperative data analysis, and (5) regulatory agency audits. No finalized surgical case nursing EMR will capture patient charges or be moved for analytical analysis until all data QCC rules are met. All violations of data QCC rules are documented, reconciled, corrected, and nursing EMR training is 
directed as needed. Furthermore, the embedded QCC process is flexible to adjust to changes within the perioperative process as needed. Moreover, the BPM framework within the non-finalized case EMR reconciliation allows QCC process improvement opportunity as well as organizational learning. Data integrity and quality within the CSIS data mart provides analytical opportunities to improve clinical effectiveness as well as process performance. Ensuring data integrity and quality within the CSIS data mart encourages trust from data consumers like knowledge workers and other stakeholders within the perioperative process as well as downstream. Likewise, ensured data integrity and quality in perioperative care documentation meets external regulatory requirements as well as minimizes audit risk. To this end, an embedded QCC process within integrated hospital information systems provides a solid foundation on which to develop and enhance BPM.

Our case study contributes to the healthcare IT literature by examining how flexible data QCC rules, an embedded QCC process, and rule violation reconciliation within a BPM framework is applicable to the hospital environment. This study prescribes an a priori framework to embed data quality into perioperative patient care documentation and foster the occurrence. Additionally, this paper also fills a gap in the literature by describing how hospital process data is both a performance measure, a management tool, and a valued resource.

This study was limited to a single case, where future research should broaden the focus to address this issue along with others that the authors may have inadvertently overlooked. The case examples presented in this study can serve as momentum for healthcare process management methodology, comprehension, and extension. The study's results should be viewed as exploratory and in need of further confirmation. Researchers may choose to further or expand the investigation; while practitioners may apply the findings and create their own version of embedded data integrity and quality within integrated hospital information systems.

\section{References}

[0] Ackoff, R. L. 1967. "Management misinformation systems," Management Science, 14(4), 147 - 156.

[1] Aiken, L., Cimiotti, J., Sloan, D., et al. 2011. "The effects of nurse staffing and nurse education on patient deaths in hospitals with different nurse work environments," Medical Care (49:12), 1047-1053.

[2] Arthur, M. \& Odo, M., 2010. "The practice of anesthesiology and perioperative care," AMWA Journal (25:2), 50-56.

[3] Beverland, M. \& Lindgreen, A, 2010. "What makes a good case study? A positivist review of qualitative case research," Industrial Marketing Management (39:1), 56-63.
[4] Blumenthal, D. 2012. "Performance improvement in healthcare-seizing the moment," NEJOM (366:21), pp. 1953-1955.

[5] CMS 2005. "Pay-for-performance quality incentives," Centers for Medicare/Medicaid Services, 10. http://www.cms.gov/Regulations-and-Guidance/Guid ance/FACA/downloads/tab_H.pdf, accessed 5/15/2017.

[6] CMS 2010. "Medicare hospital quality chartbook: 2010 performance report on outcomes measures," Centers for Medicare/Medicaid Services, 44 https://www.cms.gov/Medicare/Quality-Initiatives-PatientAssessment-Instruments/HospitalQualityInits/ OutcomeMeasures.html, accessed 5/15/2017.

[7] CMS 2014. "Meaningful Use," Centers for Medicare \& Medicaid Services, pp. 1. https://www.cms.gov/ Regulations-and-Guidance/Legislation/EHRIncentive Programs, accessed 5/15/2017.

[8] CMS 2015. "Hospital-Acquired Conditions List 2015," Centers for Medicare/Medicaid Services, pp.3 http://www.cms.gov/medicare/medicare-fee-for-servicepayment/hospitalacqcond/hospitalacquired_conditions.html. Accessed 5/15/2017.

[9] CMS 2016. "Recovery auditing in Medicare fee forservice for fiscal year 2015," Centers for Medicare/ Medicaid Services, pp. 36, https://www.cms.gov/Res earchStatistics-Data-and-Systems/Monitoring-Program s/Medicare-FFS-Compliance-Programs/Recovery-AuditProgram/Downloads/FY2015-Medicare-FFS-RAC-Reportto-Congress.pdf, accessed 5/15/2017.

[10] DeBroux, A. \& Reed, C. 2015. "How to build trust in your data warehouse," Business Intelligence Journal, (20:1), pp. 50-55.

[11] Dougherty, D. and Conway, P., 2008. "The 3Ts road map to transform US health care," JAMA, 299(19), 23192321.

[12] Eichhorn, R., 2013. "Practical current issues in perioperative patient safety," Canadian Journal of Anesthesia (60:1), 2013, 111-118.

[13] Eisenhardt, K. 1989. "Building theories from case study research", Academy of Management Review (14: 4), 532-550.

[14] Fowler, P., Craig, J., \& Fredendall, L., 2008. "Perioperative workflow: Barriers to efficiency, risks, and satisfaction," AORN Journal (87:1), 187-208.

[15] Gertz, M. 1998. "Managing data quality and integrity in federated databases," Proceedings of the IFIP TC11 Working Group 11.5, 2nd Working Conference on Integrity and Internal Control in IS: Bridging Business Requirements and Research Results, $211-230$.

[16] Jones S., Rudin R., Perry T., \& Shekelle P. 2014. "Health Information Technology: An Updated Systematic Review with a Focus on Meaningful Use," Annuals of Internal Medicine (160:1), 48-54.

[17] Herzer, K., Mark, L., Michelson, J., Saletnik, L., and Lundquist, C. 2008. "Designing and implementing a comprehensive quality and patient safety management 
model: a paradigm for perioperative improvement," Journal of Patient Safety (4:2), 84 - 92.

[18] Jeston, J. \& Nelis, J. 2008. Business Process Management: Practical Guidelines to Successful Implementations, Second Edition. Burlington, MA: Elsevier, Ltd.

[19] Kanich, D. \& Byrd, J. 1996. "How to increase efficiency in the operating room," Surgical Clinics of North America (76:1), 161 - 173.

[20] Karimi, J., 1988. "Strategic planning for information systems: requirements and information engineering methods", Journal of MIS (4:4), 5-24.

[21] Lee, Y. \& Strong, D. 2003. "Knowing why about data processes and data quality," Journal of MIS (20:3), 13 - 39.

[22] Lee, Y., Pipino, L., Strong, D., \& Wang, R. 2004. "Process embedded data integrity," Journal of Database Management (5:1), 87-103.

[23] Marjamaa, R., Vakkuri, A., \& Kirvela, O. 2008. "Operating room management: Why, how and by whom?" ActaAnaesthesiol Scandanavia (52:5), 596-600.

[24] Munroe, M. \& Wheeler, B. 1980. "Planning, critical success factors, and management's information requirements," MIS Quarterly (4:4), 27 - 37.

[25] NQF 2008. "Serious reportable events," National Quality Forum, 4. http://www.qualityforum.org/Publica tions/2008/10/Serious_Reportable_Events.aspx, accessed $5 / 15 / 2017$.

[26] Paré, G. 2001. "Using a positivist case study methodology to build and test theories in information systems," HEC Montréal Business School, Montréal, Canada. http://expertise.hec.ca/gresi/wpcontent/uploads/ 2013/02/cahier0109.pdf, accessed 04/28/2016.

[27] Peters, J. \& Blasco, T. 2004. "Enhancing hospital performance through perioperative services," Physician Executive (30:6), 26-31.

[28] Pirie, S., 2011. "Documentation \& record keeping," Journal of Perioperative Practice (21:1), 22-27.

[29] PwC Health Research Institute 2012. "The future of the academic medical center: Strategies to avoid a meltdown," PwC LLP. http://www.pwc.com/us/en/ healthindustries/publications/the-future-of-academic-medicalcenters.jhtml, accessed 04/28/2016.

[30] Rockart, J., 1979. "Chief executives define their own data needs," Harvard Business Review (57:2), 81-93.

[31] Rothschild, J., 2004. "Computerized physician order entry in the critical care and general inpatient setting: a narrative review," Journal of Critical Care (19:4), 271-278.

[32] Ryan, J., Doster, B., Daily, S., \& Lewis, C., 2014. "A balanced perspective to perioperative process management aligned to hospital strategy," International Journal of Healthcare Information Systems \& Informatics (IJHISI) (9:4), 1-19.

[33] Ryan, J., Doster, B., Daily, S., \& Lewis, C., 2016. "A case study perspective for balanced perioperative workflow achievement through data-driven process improvement," IJHISI (11:3), 19-41.
[34] Ryan, J., Doster, B., Daily, S., \& Lewis, C., 2017. "Using key performance indicators to reduce perceived complexity and improve patient workflow," IJHSI (12:4), 13-30.

[35] Schnall, R., Cook, S., et al. 2012. "Patient safety issues in advanced nursing students' care settings," Journal of Nursing Care Quality (27:2), 132-138.

[36] Silverman, D. \& Rosenbaum, S., 2009. "Integrated assessment and consultation for the preoperative patient," Medical Clinics of North America (93), 963-977.

[37] Strong, D., Lee, Y., \& Wang, R., 1997. "Data quality in context," Comm of the ACM (40:5), 103-110.

[38] Tarantino, D. 2003. "Process redesign part 1: Process selection," Physician Executive (29:6), 71-73.

[39] Tenner, A. \& DeToro, I. 1997. Process redesign: the implementation guide for managers. Upper Saddle River, NJ: Prentice-Hall, Inc.

[40] Turban, E.; Sharda, R.; Aronson, J.; \& King, D. 2008. Business Intelligence: A managerial approach. Upper Saddle River, New Jersey: Prentice Hall

[41] Utzolino, S., Kaffarnik, M., Keck, et al. 2010. "Unplanned discharges from a SICU: readmissions and mortality," Journal of Critical Care (25:1), 375-381.

[42] van Deursen, A., 1999. "Software renovation", ERCIM News (36:1), 13-14.

[43] Walton, M. 1986. The Deming Management Method. New York: Dodd, Mead.

[44] Waters T., Daniels M., Bazzoli G., et al. 2015. "Effect of Medicare's nonpayment for hospital-acquired conditions: lessons for future policy," JAMA Internal Medicine (175:3), $347-354$.

[45] Weber, R., 2004. "The rhetoric of positivism versus interpretivism: a personal view," MIS Quarterly, 28(1): iiixii.

[46] Wears, R. \& Berg, M. 2005. "Computer technology and clinical work: still waiting for Godot", JAMA (293:10), 1261-1263.

[47] Weissman, C. \& Klein, N. 2008. "The importance of differentiating between elective and emergency postoperative critical care patients," Journal of Critical Care (23), 308-316.

[48] Weiskopf, N. \& Weng, C. 2013. "Methods and dimensions of electronic health record data quality assessment: enabling reuse for clinical research," JAMIA (20:1), 141-155

[49] Wright, J., Roche, A., \& Khoury, A., 2010. "Improving on-time surgical starts in an operating room," Canadian Journal of Surgery (53:3), 167-170.

[50] Yin, R. K., 2003. Case study research: Design and methods, Third Edition. Thousand Oaks, California: Sage Publications.

[51] Zani, W. M. 1970. "Blueprint for MIS," Harvard Business Review (48:6), 85 - 90. 